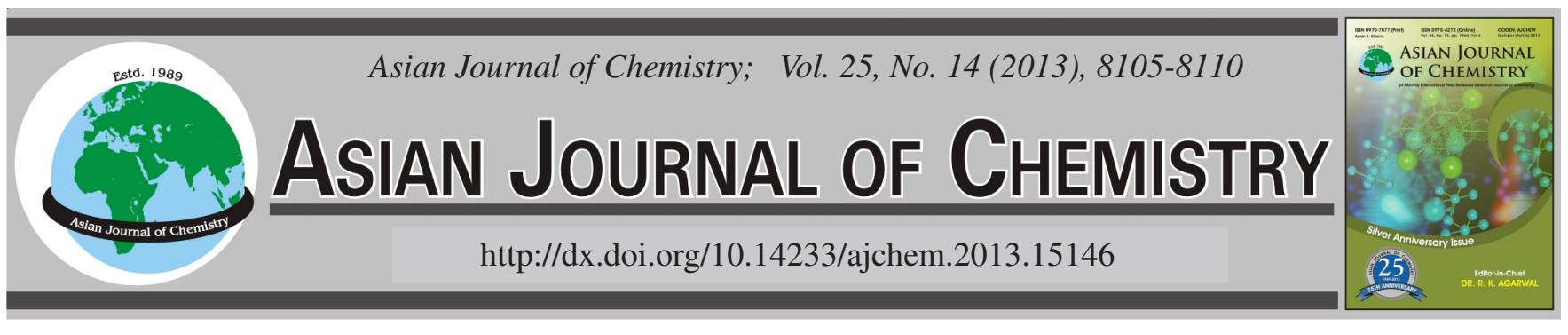

\title{
Synthesis and Spectral Studies of Acetophenone Schiff Bases and Evaluation of their Antimicrobial Activities
}

\author{
Hira Mir, Dildar Ahmed* and Hafiz Muhammad Adeel Sharif
}

Department of Chemistry, Forman Christian College (A Chartered University), Ferozpur Road, Lahore, Pakistan

*Corresponding author: E-mail: dildarahmed@fccollege.edu.pk

Seven acetophenone derived Schiff bases with different amines were synthesized including, (Z)-2-[(1-phenylethylidene)amino]benzoic acid $\left(\mathbf{H L}^{1}\right),(\mathrm{Z})-4-\left[(1-p h e n y l e t h y l i d e n e)\right.$ amino]benzoic acid $\left(\mathbf{H L}^{2}\right),(\mathrm{E})-\mathrm{N}-\left(1-\right.$ phenylethylidene)naphthalen-2-amine $\left(\mathbf{H L}^{3}\right),(\mathrm{E})-1$-phenyl-2(1-phenylethylidene)hydrazine $\left(\mathbf{H L}^{4}\right), \mathrm{N}^{1}, \mathrm{~N}^{2}$-bis(1-phenylethylidene)ethane-1,2-diamine $\left(\mathbf{H L}^{5}\right), \mathrm{N}^{1}(\mathrm{Z}), \mathrm{N}^{2}(\mathrm{Z})-\mathrm{N}^{1}, \mathrm{~N}^{2}-$ bis $(1-$ phenylethylidene)benzene-1,2-diamine $\left(\mathbf{H L}^{6}\right)$ and $\mathrm{N}^{1}, \mathrm{~N}^{4}$-bis (1-phenylethylidene)benzene-1,4-diamine $\left(\mathbf{H L}^{7}\right)$ and characterized by spectral studies (IR, MS and NMR) and elemental analysis. Their antimicrobial activity was extensively investigated against 20 Gram-negative and 10 Gram-positive bacterial strains. Schiff bases $\mathbf{H L}^{1-5}$ showed good antibacterial activity against almost all bacterial strains. $\mathbf{H L}^{3}$ showed remarkable activity against Enterococcus sp., Citrobacter freundii, Salmonella typhi and Pseudomonas aurantiaca. $\mathbf{H L}^{4}$ gave incredible inhibition against Enterobacter aerogenes. HL $^{7}$ demonstrated notable activity against Staphylococcus aureus. (E)-N-(1phenylethylidene)naphthalen-2-amine $\left(\mathbf{H L}^{3}\right)$ is reported for the first time.

Key Words: Acetophenone, Schiff bases, Spectroscopic, Antimicrobial activity.

\section{INTRODUCTION}

Growing pathogenic resistance to existing antibiotic drugs is one of the most baffling challenges to human health in the modern times and necessitates constant efforts to discover more effective and safer therapeutic agents ${ }^{1-3}$. The infectious diseases are, in fact, one of the main causes of deaths the world over ${ }^{4}$. The screening of both the natural products and the synthetic compounds against different pathogenic microorganisms has thus emerged to be one of the most active fields of research today.

The Schiff bases ${ }^{5-8}$, or substances with azomethine functionality, comprise a major class of organic compounds being investigated for multifarious applications including their possible development into therapeutic agents. They have been found to possess antibacterial ${ }^{9,10}$, antifungal ${ }^{11}$, antiviral ${ }^{12}$, antiinflammatory ${ }^{13}$, analgesic ${ }^{14}$, anti- tumour ${ }^{15}$, anticonvulsant ${ }^{16}$, anti $\mathrm{HIV}^{17}$ and antileishmanial ${ }^{18}$ activities. They are also good ligands ${ }^{19}$ and can coordinate with metals in vitro as well as in vivo.

Acetophenone, a ketone having both an aliphatic and an aromatic moiety, has been shown to form Schiff bases exhibiting considerable antibacterial ${ }^{20,21}$ and antifungal ${ }^{22,23}$ activities along with other useful applications ${ }^{24-28}$.

As part of our ongoing search for new therapeutic agents, seven different Schiff bases were synthesized from unsubstituted acetophenone and different primary amines including monoand diamines of both aromatic and aliphatic nature. According to our knowledge, acetophenone derived Schiff base with $\beta$ naphthylamine is being reported for the first time. Spectral studies including ${ }^{1} \mathrm{H}$ NMR, ${ }^{13} \mathrm{C}$ NMR, IR and MS were carried out to characterize the compounds. Synthesised Schiff bases were then screened for the first time ever against thirty different bacterial strains including gram positive and gram negative bacteria, pathogenic as well as non-pathogenic.

\section{EXPERIMENTAL}

All the reagents and solvents were of analytical-grade quality purchased from Aldrich Co and were used without further purification. Melting points were found on Gallenkamp (Electronic) melting point apparatus and were uncorrected. The reactions were monitored by thin-layer chromatography (TLC) on silica gel plates (Sigma-Aldrich). A mixture of ethanol and ethyl acetate (1:1) was employed as mobile phase and UV lamp, Model UVLS-225 D, was used for detection.

The IR spectra of the compounds were taken using $\mathrm{KBr}$ disks on Varian 640-IR spectrometer $\left(\mathrm{cm}^{-1}\right)$. UV-VIS absorption spectra were recorded with Spectro UV-VIS Double PC 8 Auto Cell with variable bandwidth of $0.5,1.0,2.0$ and 5.0 nm, Model UVD-3200 spectrometer. Both ${ }^{1} \mathrm{H}$ and ${ }^{13} \mathrm{C}$ NMR spectra were recorded on a Bruker $300 \mathrm{MHz}$ NMR spectrometer 
(H.E.J. Research Institute of Chemistry, Karachi, Pakistan), with chemical shift in ppm downfield from TMS as an internal reference. High resolution mass spectra were captured on Varian MAT 312 mass spectrometer (H.E.J. Research Institute of Chemistry, Karachi, Pakistan).

\section{Synthesis of Schiff bases}

Schiff bases $\mathbf{H L}^{\mathbf{1}}$ to $\mathbf{H L}^{\mathbf{4}}$ : Acetophenone $(1.16 \mathrm{~mL}, 10$ mmol) with $o$-aminobenzoic acid $\left(1.37 \mathrm{~g}, 10 \mathrm{mmol}\right.$ ) (for $\mathbf{H L}^{\mathbf{1}}$ ), $p$-aminobenzoic acid $\left(1.37 \mathrm{~g}, 10 \mathrm{mmol}\right.$ ) (for $\left.\mathbf{H L}^{2}\right), \beta$-naphthylamine (1.43 g, $10 \mathrm{mmol}$ ) (for $\mathbf{H L}^{3}$ ) and phenylhydrazine $\left(0.99 \mathrm{~mL}, 10 \mathrm{mmol}\right.$ ) (for $\left.\mathbf{H L}^{4}\right)$ in different round bottom flasks were refluxed in $20 \mathrm{~mL}$ methanol at $40^{\circ} \mathrm{C}$ for $3 \mathrm{~h}$ in the presence of $1 \mathrm{~mL}$ glacial acetic acid. In each case, the precipitated base was filtered off, recrystallized from absolute ethanol and dried in vacuum desiccator. The structure of the synthesized schiff bases are given in Fig. 1.
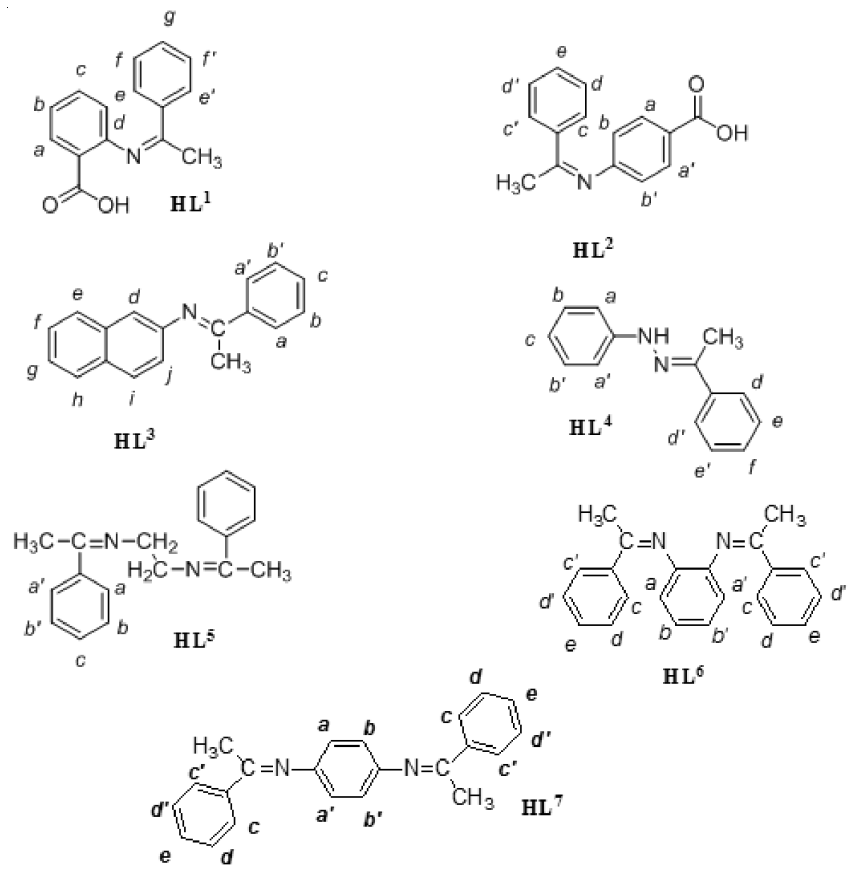
$\mathrm{HL}^{6}$

Fig. 1. Structures of synthesized Schiff bases

Schiff bases $\mathbf{H L}^{5}$ to $\mathbf{H L}^{7}$ : In different experiments, acetophenone $(2.33 \mathrm{~mL}, 20 \mathrm{mmol})$ with ethylene diamine $(0.66$ $\mathrm{mL}, 10 \mathrm{mmol}$ ) (for $\mathbf{H L}^{5}$ ), $o$-amino aniline (1.08 g, $10 \mathrm{mmol}$ ) (for $\mathbf{H L}^{6}$ ) and $p$-amino aniline (1.08 g, $10 \mathrm{mmol}$ ) (for $\mathbf{H L}^{7}$ ) was refluxed at $40{ }^{\circ} \mathrm{C}$ for $3 \mathrm{~h}$ in $20 \mathrm{~mL}$ of methanol in the presence of $1 \mathrm{~mL}$ glacial acetic acid. The resultant ligands were filtered, recrystallized from absolute ethanol and dried in vacuum desiccator. The structures of proposed ligands are shown in Fig. 1.

\section{Antimicrobial analysis}

Test microbes: Seventeen different bacterial cultures were acquired from The Children Hospital, Lahore, Pakistan including two strains of E. coli [Escherichia coli (1) and Escherichia coli (2)], two strains of S. aureus [Staphylococcus aureus (1) and Staphylococcus aureus (2)], two strains of $P$. aeruginosa [Pseudomonas aeruginosa (1) and Pseudomonas aeruginosa (2)], two unidentified Bacillus species [Bacillus sp.(1) and Bacillus sp.(2)] and one strain each of Pseudomonas aurantiaca , Salmonella typhi (1), Stenotroph maltophilia, Enterobacter cloacae, Enterobacter aerogenes, Klebsiella pneumoniae, Staphylococcus epidermidis, Bacillus subtilis and Bacillus megaterium. Thirteen different microbial colonies were attained from biotechnology laboratory, Forman Christian College, Lahore, Pakistan including two strains of E. coli [Escherichia coli (3) and Escherichia coli (4)] and single strain each of Pseudomonas aeruginosa (3), Pseudomonas sp., Salmonella typhi (2), Salmonella sp., Achromobacter xylosoxidans, Azospirillum lipoferum, Rhizobium sp., Citrobacter freundii, Staphylococcus aureus (3), Bacillus sp.(3) and Enterococcus sp. All strains were stored at $-20^{\circ} \mathrm{C}$ until utilized.

Determination of zones of inhibition (ZI): Agar well diffusion method ${ }^{29,30}$ was employed for antimicrobial screening in terms of the zones of inhibition.

Preparation of standard dilutions: In each case, $2 \mathrm{mg}$ of a synthesized compound was dissolved per $\mathrm{mL}$ of DMSO to yield stock solution, which was serially diluted to $0.5-1$ $\mathrm{mg} / \mathrm{mL}$. Three antibiotic drugs levofloxacin, cefixime and amoxylin were used as standard with final concentration of 2 $\mathrm{mg} / \mathrm{mL}$ in DMSO.

Preparation of inoculums: All microbial cultures were re-isolated thrice successively on Mueller-Hinton Agar, MHA (Merck) with incubation period of $24 \mathrm{~h}$ at $37^{\circ} \mathrm{C}$ and identity confirmed by standard bacteriological methods. A loop full of bacterial suspension was then diluted with sterile physiological solution to standardize inoculum density up to $10^{8} \mathrm{CFU} /$ $\mathrm{mL}$ of bacterial cells (equivalent to turbidity of McFarland, barium sulphate standard 0.5 ) followed by $24 \mathrm{~h}$ incubation at $37^{\circ} \mathrm{C}$.

Antimicrobial screening (zones of inhibition): The bacterial inoculums were uniformly swabbed on prepared Mueller-Hinton Agar plate followed by a session of drying, after which three wells of $7 \mathrm{~mm}$ diameter each were dug in the agar gel $33 \mathrm{~mm}$ apart from one another using a sterile cork borer. A $100 \mu \mathrm{L}$ volume of test compound dilutions were pipetted into the triplicate wells and allowed to stand for an hour for diffusion to take place. Finally, plates were incubated at $37^{\circ} \mathrm{C}$ for $24 \mathrm{~h}$ after which zones of inhibition were recorded to the nearest $\mathrm{mm}$.

Determination of minimum inhibitory concentration (MIC): Minimum inhibitory concentration (MIC) is defined as the lowest concentration of an anti-microbial agent requisite to inhibit bacterial growth. The agar dilution method ${ }^{31,32}$ was executed to evaluate MICs of synthesized compounds and antibiotics. Briefly, $0.004 \mathrm{~g} / \mathrm{mL}$ stock solution of each of the test compounds was prepared in DMSO, from which graded concentrations were made to achieve final concentrations (mg/ $\mathrm{mL}$ ) of $0.02,0.04,0.08,0.12,0.16,0.20,0.24,0.28,0.32$, 0.36 and 0.40 in total $20 \mathrm{~mL}$ of Mueller-Hinton Agar plates by varying the volume of agar and test stock solutions. The plates were spotted with $0.1 \mathrm{~mL}$ overnight activated cultures of the microbes and incubated at $37^{\circ} \mathrm{C}$ for $24 \mathrm{~h}$.

\section{RESULTS AND DISCUSSION}

In the present study, seven different Schiff bases of the ketone acetophenone were synthesized with different amines using the conventional reflux method. The structures (Fig. 1) 
of the bases were elucidated on the bases of elemental analysis and spectroscopic data. The bases were then subjected to antimicrobial screening against different bacterial strains.

(Z)-2-((1-Phenylethylidene)amino)benzoic acid (HL $\left.{ }^{\mathbf{1}}\right)$ : Orange yellow; yield (\%): 86; m.p. $233{ }^{\circ} \mathrm{C}$; anal. calcd. (\%) for $\mathrm{C}_{15} \mathrm{H}_{13} \mathrm{NO}_{2}$ : 75.31, C; 5.43, H; 5.85, N; 13.38, O. Found (\%): 75.30, C; 5.42, H; 5.83, N; 13.35, O. Selected IR (KBr, $\left.V_{\max }, \mathrm{cm}^{-1}\right) 1627(-\mathrm{C}=\mathrm{N}-), 2900-3100(\mathrm{O}-\mathrm{H}), 1721(\mathrm{C}=\mathrm{O}), 1290$ (C-O). ${ }^{1} \mathrm{H}$ NMR $\left(\mathrm{CD}_{3} \mathrm{OD}, 300 \mathrm{MHz}\right) \delta 2.28\left(\mathrm{~s}, 3 \mathrm{H}, \mathrm{CH}_{3}\right), 6.82$ $\left(\mathrm{m}, 3 \mathrm{H}, \mathrm{ArH}_{\mathrm{f}=\mathrm{f}^{\prime}, \mathrm{g}}\right), 7.25\left(\mathrm{~d}, 1 \mathrm{H}, \mathrm{ArH}_{\mathrm{d}},{ }^{3} J=7.2 \mathrm{~Hz}\right), 7.52(\mathrm{t}, 1 \mathrm{H}$, $\left.\mathrm{ArH}_{\mathrm{c}},{ }^{3} J=7.2 \mathrm{~Hz}\right), 7.74\left(\mathrm{~d}, 2 \mathrm{H}, \mathrm{ArH}_{\mathrm{e}=\mathrm{e}},{ }^{3} J=7.5 \mathrm{~Hz}\right), 7.85(\mathrm{t}$, $\left.1 \mathrm{H}, \mathrm{ArH}_{\mathrm{b}},{ }^{3} J=7.2 \mathrm{~Hz}\right), 8.12\left(\mathrm{~d}, 1 \mathrm{H}, \mathrm{ArH}_{\mathrm{a}},{ }^{3} \mathrm{~J}=7.2 \mathrm{~Hz}\right), 11.9(\mathrm{~s}$, $1 \mathrm{H}, \mathrm{COOH}) ;{ }^{13} \mathrm{C} \mathrm{NMR}\left(\mathrm{CD}_{3} \mathrm{OD}, 75 \mathrm{MHz}\right) \delta 18.3\left(1 \mathrm{C}, \mathrm{CH}_{3}\right)$, 111.2 (1C), 122.3 (1C), 125.0 (1C), 126.7 (3C), 127.8 (1C), 133.1 (1C), 135.2 (1C), 137.8 (1C), 150.2 (1C, C-N), 165.3 $(1 \mathrm{C}, \mathrm{C}=\mathrm{N}), 171.0(1 \mathrm{C}, \mathrm{C}=\mathrm{O}) ; \mathrm{MS}: \mathrm{m} / \mathrm{z} 239\left[\mathrm{M}^{+}\right]$.

(Z)-4-((1-Phenylethylidene)amino)benzoic acid $\left(\mathbf{H L}^{2}\right)$ : Lime yellow; yield (\%): 82; m.p. $227^{\circ} \mathrm{C}$; anal. calcd. (\%) for $\mathrm{C}_{15} \mathrm{H}_{13} \mathrm{NO}_{2}$ : 75.31, C; 5.43, H; 5.85, N; 13.38, O. Found (\%): 75.31, C; 5.42, H; 5.84, N; 13.36, O. Selected IR (KBr, $v_{\max }$, $\left.\mathrm{cm}^{-1}\right): 1629(-\mathrm{C}=\mathrm{N}-), 2900-3100(\mathrm{O}-\mathrm{H}), 1720(\mathrm{C}=\mathrm{O}), 1293$ $(\mathrm{C}-\mathrm{O}) .{ }^{1} \mathrm{H} \mathrm{NMR}\left(\mathrm{CD}_{3} \mathrm{OD}, 300 \mathrm{MHz}\right) \delta 2.27\left(\mathrm{~s}, 3 \mathrm{H}, \mathrm{CH}_{3}\right), 6.86$ $\left(\mathrm{m}, 3 \mathrm{H}, \mathrm{ArH}_{\mathrm{d}=\mathrm{d}^{\prime}, \mathrm{e}}\right), 7.34\left(\mathrm{~d}, 2 \mathrm{H}, \mathrm{ArH}_{\mathrm{b}=\mathrm{b}},{ }^{3} J=7.5 \mathrm{~Hz}\right), 7.52(\mathrm{~d}$, $\left.2 \mathrm{H}, \mathrm{ArH}_{\mathrm{c}=\mathrm{c}^{\prime}},{ }^{3} J=7.5 \mathrm{~Hz}\right), 8.0\left(\mathrm{~d}, 2 \mathrm{H}, \mathrm{ArH}_{\mathrm{a}=\mathrm{a}^{\mathrm{a}}},{ }^{3} J=7.5 \mathrm{~Hz}\right), 11.8$ $(\mathrm{s}, 1 \mathrm{H}, \mathrm{COOH}) ;{ }^{13} \mathrm{C} \mathrm{NMR}\left(\mathrm{CD}_{3} \mathrm{OD}, 75 \mathrm{MHz}\right) \delta 24.2\left(1 \mathrm{C}, \mathrm{CH}_{3}\right)$, 112.2 (2C), 120.3(2C), 126.0(1C), 130.8(2C), 133.1(2C), 135.4(4C), 137.8(1C), 152.1(1C, C-N), 163.4(1C, C=N), 171.1(1C, C=O); MS: m/z $239\left[\mathrm{M}^{+}\right]$.

(E)-N-(1-Phenylethylidene)naphthalen-2-amine $\left(\mathrm{HL}^{3}\right)$ : Faded pink; yield (\%): 84; m.p. $239^{\circ} \mathrm{C}$; anal. calcd. (\%) for $\mathrm{C}_{18} \mathrm{H}_{15} \mathrm{~N}$ : 88.16, C; 6.12, H; 5.72, N. Found (\%): 88.08, C; 6.11, H; 5.71, N. Selected IR (KBr, $\left.\nu_{\max }, \mathrm{cm}^{-1}\right)$ : $1631(-\mathrm{C}=\mathrm{N}-)$. ${ }^{1} \mathrm{H}$ NMR $\left(\mathrm{CD}_{3} \mathrm{OD}, 300 \mathrm{MHz}\right) \delta 2.28\left(\mathrm{~s}, 3 \mathrm{H}, \mathrm{CH}_{3}\right), 7.23(\mathrm{~d}, 1 \mathrm{H}$, $\left.\mathrm{ArH}_{\mathrm{j}},{ }^{3} \mathrm{~J}=7.2 \mathrm{~Hz}\right), 7.32\left(\mathrm{~m}, 3 \mathrm{H}, \mathrm{ArH}_{\mathrm{b}=\mathrm{b}}, \mathrm{c}\right), 7.42\left(\mathrm{~m}, 2 \mathrm{H}, \mathrm{ArH}_{\mathrm{g}, \mathrm{f}}\right)$, 7.72(d, 2H, $\left.\mathrm{ArH}_{\mathrm{a}=\mathrm{a}^{\prime}}\right), 7.88\left(\mathrm{~m}, 4 \mathrm{H}, \mathrm{ArH}_{\mathrm{d}, \mathrm{e}, \mathrm{h}, \mathrm{i}}\right) ;{ }^{13} \mathrm{C} \mathrm{NMR}\left(\mathrm{CD}_{3} \mathrm{OD}\right.$, $75 \mathrm{MHz}) \delta 18.3\left(1 \mathrm{C}, \mathrm{CH}_{3}\right), 110.2(1 \mathrm{C}), 115.3(1 \mathrm{C}), 120.0(2 \mathrm{C})$, 123.3(1C), 125.1(2C), 125.3(2C), 125.6(2C), 126.2(1C), 127.4(1C), 128.2(1C), 134.2(1C), 135.2(1C, C-N), 146.3(1C, $\mathrm{C}=\mathrm{N}) ; \mathrm{MS}: \mathrm{m} / \mathrm{z} 245\left[\mathrm{M}^{+}\right]$.

(E)-1-Phenyl-2-(1-phenylethylidene)hydrazine $\left(\mathrm{HL}^{4}\right)$ : Chocolate brown; yield (\%): 90; m.p. $64{ }^{\circ} \mathrm{C}$; anal. calcd. (\%) for $\mathrm{C}_{14} \mathrm{H}_{14} \mathrm{~N}_{2}$ : 80.00, C; 6.66, H; 13.37, N. Found (\%): 79.92, C; 6.64, H; 13.34, N. Selected IR $\left(\mathrm{KBr}, v_{\max }, \mathrm{cm}^{-1}\right): 1637$ $(-\mathrm{C}=\mathrm{N}-), 3350(\mathrm{NH}) .{ }^{1} \mathrm{H} \mathrm{NMR}\left(\mathrm{CD}_{3} \mathrm{OD}, 300 \mathrm{MHz}\right) \delta 2.28(\mathrm{~s}$, $\left.3 \mathrm{H}, \mathrm{CH}_{3}\right), 6.68\left(\mathrm{t}, 1 \mathrm{H}, \mathrm{Ar}_{c}\right), 7.27\left(\mathrm{~m}, 3 \mathrm{H}, \mathrm{ArH}_{\mathrm{b}=\mathrm{b}^{\prime}, \mathrm{c}}\right), 7.02(\mathrm{t}, 2 \mathrm{H}$, $\left.\mathrm{ArH}_{\mathrm{b}=\mathrm{b}},{ }^{3} J=7.2 \mathrm{~Hz}\right), 7.25\left(\mathrm{~d}, 2 \mathrm{H}, \mathrm{ArH}_{\mathrm{a}=\mathrm{a}},{ }^{3} J=7.5 \mathrm{~Hz}\right), 7.52$ (t, 3H, $\left.\mathrm{ArH}_{\mathrm{e}=\mathrm{e}^{\prime}, \mathrm{f}}\right), 12.96(\mathrm{~s}, 1 \mathrm{H}, \mathrm{NH}) ;{ }^{13} \mathrm{C} \mathrm{NMR}\left(\mathrm{CD}_{3} \mathrm{OD}, 75\right.$ $\mathrm{MHz}) \delta 18.3\left(1 \mathrm{C}, \mathrm{CH}_{3}\right), 115.2(2 \mathrm{C}), 115.4(1 \mathrm{C}) 116.5 .2(2 \mathrm{C})$, 116.9 (2C), 118.6 (2C), 136.6 (1C), 138.7 (1C), 168.2 (1C, $\mathrm{C}=\mathrm{N}) ; \mathrm{MS}: \mathrm{m} / \mathrm{z} 210\left[\mathrm{M}^{+}\right]$.

$\mathrm{N}^{1}, \mathbf{N}^{2}$-Bis(1-phenylethylidene)ethane-1,2-diamine $\left(\mathbf{H L}^{5}\right)$ : Pale yellow; yield (\%): 80; m.p. $120^{\circ} \mathrm{C}$; anal. calcd. (\%) for $\mathrm{C}_{18} \mathrm{H}_{20} \mathrm{~N}_{2}: 81.20, \mathrm{C} ; 7.15, \mathrm{H} ; 10.17, \mathrm{~N}$. Found $(\%)$ : 81.81, C; 7.57, H; 10.60, N. Selected IR $\left(\mathrm{KBr}, v_{\max }, \mathrm{cm}^{-1}\right)$ : 1635 (-C=N-). ${ }^{1} \mathrm{H}$ NMR $\left(\mathrm{CD}_{3} \mathrm{OD}, 300 \mathrm{MHz}\right) \delta 2.26(\mathrm{~s}, 6 \mathrm{H}$, $\left.\mathrm{CH}_{3}\right), 4.24$ (s, 2H, $-\mathrm{CH}_{2}$ ) $, 7.27\left(\mathrm{~m}, 6 \mathrm{H}, \mathrm{ArH}_{\mathrm{b}=\mathrm{b}^{\prime}, \mathrm{c}}\right), 7.54(\mathrm{~d}, 4 \mathrm{H}$, $\left.\mathrm{ArH}_{\mathrm{a}=\mathrm{a}^{\prime}},{ }^{3} J=7.2 \mathrm{~Hz}\right) ;{ }^{13} \mathrm{C} \mathrm{NMR}\left(\mathrm{CD}_{3} \mathrm{OD}, 75 \mathrm{MHz}\right) \delta 18.0(2 \mathrm{C}$, $\left.\mathrm{CH}_{3}\right), 54.1$ (2C, $\left.-\mathrm{CH}_{2}-\right), 127.2$ (4C), 128.1 (6C), 128.6 (2C), 142.7 (2C), $168.3(2 \mathrm{C}, \mathrm{C}=\mathrm{N}) ; \mathrm{MS}: \mathrm{m} / \mathrm{z} 264\left[\mathrm{M}^{+}\right]$.

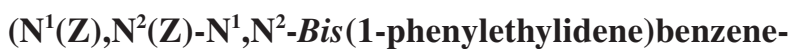
1,2-diamine $\left(\mathbf{H L}^{6}\right)$ : Earth brown; yield (\%): 88; m.p. $238{ }^{\circ} \mathrm{C}$; anal. calcd. (\%) for $\mathrm{C}_{22} \mathrm{H}_{20} \mathrm{~N}_{2}: 84.61, \mathrm{C} ; 6.41, \mathrm{H} ; 8.98$, N. Found (\%): 84.58, C; 6.40, H; 8.97, N. Selected IR (KBr, $\left.\mathrm{v}_{\max }, \mathrm{cm}^{-1}\right)$ : $1632(-\mathrm{C}=\mathrm{N}-) .{ }^{1} \mathrm{H}$ NMR $\left(\mathrm{CD}_{3} \mathrm{OD}, 300 \mathrm{MHz}\right) \delta 1.98(\mathrm{~s}, 6 \mathrm{H}$, $\left.\mathrm{CH}_{3}\right), 6.92-7.11\left(\mathrm{~m}, 4 \mathrm{H}, \mathrm{Ar}_{\mathrm{b}=\mathrm{b}^{\prime}, \mathrm{e}}\right), 7.15-7.30$ (m, 4H, $\mathrm{ArH}_{\mathrm{d}=\mathrm{d}^{\prime}}$ ), $7.43\left(\mathrm{~d}, 4 \mathrm{H}, \mathrm{ArH}_{\mathrm{c}=\mathrm{c}},{ }^{3} J=7.5 \mathrm{~Hz}\right), 7.59\left(\mathrm{~d}, 4 \mathrm{H}, \mathrm{ArH}_{\mathrm{a}=\mathrm{a}},{ }^{3} \mathrm{~J}=\right.$ $7.5 \mathrm{~Hz}) ;{ }^{13} \mathrm{C} \mathrm{NMR}\left(\mathrm{CD}_{3} \mathrm{OD}, 75 \mathrm{MHz}\right) \delta 22.2\left(2 \mathrm{C}, \mathrm{CH}_{3}\right), 121.9$ (2C), 122.7 (2C), 126.9 (4C), 127.7 (4C), 128.4 (2C), 129.0 (2C), 129.1 (2C), 169.7 (2C, C=N); MS: m/z 312 [M+

$\mathbf{N}^{1}, \mathbf{N}^{4}$-Bis(1-phenylethylidene)benzene-1,4-diamine $\left(\mathbf{H L}^{7}\right)$ : Earth brown; yield (\%): 88; m.p. $238^{\circ} \mathrm{C}$; anal. calcd. (\%) for $\mathrm{C}_{22} \mathrm{H}_{20} \mathrm{~N}_{2}$ : 84.61, C; 6.41, H; 8.98, N. Found (\%): 84.60, $\mathrm{C} ; 6.39, \mathrm{H} ; 8.95, \mathrm{~N}$. Selected IR $\left(\mathrm{KBr}, \mathrm{v}_{\max }, \mathrm{cm}^{-1}\right): 1631$ $(-\mathrm{C}=\mathrm{N}-) .{ }^{1} \mathrm{H}$ NMR $\left(\mathrm{CD}_{3} \mathrm{OD}, 300 \mathrm{MHz}\right) \delta 2.28\left(\mathrm{~s}, 6 \mathrm{H}, \mathrm{CH}_{3}\right)$, $6.88\left(\mathrm{~s}, 4 \mathrm{H}, \mathrm{Ar}_{\mathrm{a}=\mathrm{a}^{\prime}=\mathrm{b}=\mathrm{b}^{\prime}}\right), 7.47\left(\mathrm{~m}, 6 \mathrm{H}, \mathrm{ArH}_{\mathrm{d}=\mathrm{d}^{\prime}, \mathrm{e}}\right), 7.92(\mathrm{dd}, 4 \mathrm{H}$, $\left.\mathrm{ArH}_{\mathrm{c}=\mathrm{c}^{\prime}},{ }^{3} J=7.2 \mathrm{~Hz},{ }^{4} J=2.5 \mathrm{~Hz}\right) ;{ }^{13} \mathrm{C} \mathrm{NMR}\left(\mathrm{CD}_{3} \mathrm{OD}, 75 \mathrm{MHz}\right)$ $\delta 18.2\left(2 \mathrm{C}, \mathrm{CH}_{3}\right), 121.7(4 \mathrm{C}), 128.4(4 \mathrm{C}), 129.2(4 \mathrm{C}), 131.7(2 \mathrm{C})$, 141.0(2C), 148.5(2C), 169.8(2C, C=N); MS: m/z $312\left[\mathrm{M}^{+}\right]$.

The IR spectra of the synthesized Schiff bases showed a strong band in the region of $1610-1640 \mathrm{~cm}^{-1}$, which is characteristic of the azomethine group $(\mathrm{C}=\mathrm{N})^{33}$. Moreover, $\mathrm{O}-\mathrm{H}$ stretch in 3300-2500 $\mathrm{cm}^{-1}, \mathrm{C}=\mathrm{O}$ stretch in 1760-1690 $\mathrm{cm}^{-1}$ and C-O stretch in 1320-1210 $\mathrm{cm}^{-1}$ was noticed in $\mathbf{H L}^{1}$ and $\mathbf{H L}^{2}$, together with ${ }^{1} \mathrm{H}$ NMR signal between 11.8-12.5 ppm confirming the presence of a carboxylic group in them. ${ }^{1} \mathrm{H}$ NMR signal in 12-14 ppm and an IR band in 3400-3200 $\mathrm{cm}^{-1}$ indicating presence of $(\mathrm{NH})$ group was observed in $\mathbf{H L}^{4}$.

Antimicrobial activities: The Schiff bases in general showed moderate activity against most of the tested microbes. In some cases, however, the activity was very good while some compounds exhibited no appreciable activity against different microorganisms. Notably, bases with single azomethine group were more active than those having two azomethine functionalities (Table-1). $\mathbf{H L}^{4}$ and $\mathbf{H L}^{3}$ proved to be most versatile antimicrobial agents combating all 30 strains with medium to high lethality, while $\mathbf{H L}^{5}$ was least active. As the Table-1 displays, $\mathbf{H L}^{3}$ showed remarkable activity against Enterococcus sp., Citrobacter freundii, Salmonella typhi and Pseudomonas aurantiaca. $\mathbf{H L}^{4}$ exhibited remarkable activity against Enterobacter aerogenes, while $\mathbf{H L}^{7}$ was notably potent against Staphylococcus aureus.

$\mathbf{H L}^{1}$ was weakly active against Escherichia coli (2), Salmonella typhi (1), Staphylococcus aureus (3) and Enterococcus sp. with zones of inhibition between 10-11 mm and MIC between 200-240 $\mu \mathrm{g} / \mathrm{mL}$. It was moderately active against Escherichia coli (4), Pseudomonas aeruginosa (1) and (2), Staphylococcus aureus (2), Bacillus sp. (3), Staphylococcus epidermidis and Salmonella sp. with zones of inhibition in the range of 16-19 mm and MIC value between 120-160 $\mu \mathrm{g} / \mathrm{mL}$ (Table-2).

$\mathbf{H L}^{2}$ was moderately active against Escherichia coli (3) and (4), Pseudomonas aeruginosa (2) and (3), Pseudomonas aurantiaca, Salmonella typhi (1), Salmonella sp., Rhizobium sp., Citrobacter freundii, Staphylococcus aureus (2), Bacillus subtilis, Bacillus sp. (3) and Enterococcus sp. with zones of inhibition between 17-24 mm and MIC between $80-160 \mu \mathrm{g} / \mathrm{mL}$ (Table-2).

$\mathbf{H L}^{3}$ was weakly active against Klebsiella pneumonia and Bacillus sp. (1) with zones of inhibition of 10 and $11 \mathrm{~mm}$, 
TABLE-1

ZONES OF INHIBITION (MM) OF ACETOPHENONE DERIVED SCHIFF BASES IN THREE DIFFERENT CONCENTRATIONS AGAINST THIRTY DIFFERENT BACTERIAL STRAINS AND THEIR COMPARISON WITH THREE STANDARD ANTIBIOTICS

\begin{tabular}{|c|c|c|c|c|c|c|c|c|c|c|c|c|c|}
\hline \multirow[t]{2}{*}{ S. No. } & \multirow[t]{2}{*}{ Bacterial strains } & \multicolumn{3}{|c|}{ Antibiotics $(2 \mathrm{mg} / \mathrm{mL})$} & \multicolumn{3}{|c|}{$\mathbf{H L}^{1}(\mathrm{mg} / \mathrm{mL})$} & \multicolumn{3}{|c|}{$\mathbf{H L}^{2}(\mathrm{mg} / \mathrm{mL})$} & \multicolumn{3}{|c|}{$\mathbf{H L}^{3}(\mathrm{mg} / \mathrm{mL})$} \\
\hline & & $\mathrm{L}$ & $\mathrm{C}$ & $\mathrm{A}$ & 2 & 1 & 0.5 & 2 & 1 & 0.5 & 2 & 1 & 0.5 \\
\hline 1 & Escherichia coli (1) & 38 & 20 & 16 & - & - & - & 12 & 10 & 7 & 10 & 9 & 7 \\
\hline 2 & Escherichia coli (2) & 40 & 23 & 20 & 12 & 9 & - & 11 & 7 & - & 24 & 20 & 17 \\
\hline 3 & Escherichia coli (3) & 40 & 25 & 20 & - & - & - & 19 & 15 & 11 & 10 & 9 & 8 \\
\hline 4 & Escherichia coli (4) & 40 & 21 & 20 & 18 & 15 & 11 & 17 & 13 & 10 & 21 & 16 & 11 \\
\hline 5 & Pseudomonas aeruginosa (1) & 40 & 20 & 15 & 16 & 11 & 8 & 11 & 7 & - & 24 & 19 & 15 \\
\hline 6 & Pseudomonas aeruginosa (2) & 38 & 19 & 22 & 18 & 14 & 11 & 17 & 11 & 7 & 28 & 24 & 20 \\
\hline 7 & Pseudomonas aeruginosa (3) & 38 & 16 & 10 & 9 & 7 & - & 17 & 13 & 10 & 27 & 21 & 18 \\
\hline 8 & Pseudomonas sp. & 24 & 10 & 13 & - & - & - & 15 & 10 & 8 & 22 & 17 & 13 \\
\hline 9 & Pseudomonas aurantiaca & 32 & 20 & 12 & 7 & - & - & 20 & 17 & 14 & 33 & 28 & 23 \\
\hline 10 & Salmonella typhi (1) & 28 & 16 & 10 & 11 & 7 & - & 16 & 11 & 8 & 32 & 26 & 22 \\
\hline 11 & Salmonella typhi (2) & 32 & 15 & 10 & - & - & - & 15 & 12 & 8 & 33 & 27 & 21 \\
\hline 12 & Salmonella sp. & 33 & 16 & 17 & 18 & 13 & 9 & 20 & 17 & 13 & 29 & 24 & 19 \\
\hline 13 & Stenotroph maltophilia & 38 & 27 & 24 & - & - & - & 14 & 10 & 8 & 22 & 17 & 11 \\
\hline 14 & Enterobacter cloacae & 32 & 21 & 10 & - & - & - & 15 & 9 & 7 & 27 & 24 & 20 \\
\hline 15 & Enterobacter aerogenes & 33 & 16 & 14 & 8 & - & - & 13 & 11 & 8 & 24 & 19 & 15 \\
\hline 16 & Klebsiella pneumonia & 38 & 30 & 15 & - & - & - & 12 & 10 & 7 & 10 & 8 & - \\
\hline 17 & Achromobacter xylosoxidans & 38 & 22 & 15 & - & - & - & 13 & 9 & - & 21 & 17 & 12 \\
\hline 18 & Azospirillum lipoferum & 35 & 28 & 20 & 7 & - & - & 12 & 8 & - & 23 & 19 & 16 \\
\hline 19 & Rhizobium sp. & 30 & 16 & 15 & - & - & - & 16 & 12 & 9 & 24 & 20 & 17 \\
\hline 20 & Citrobacter freundii & 32 & 16 & 11 & 7 & - & - & 22 & 16 & 12 & 32 & 26 & 21 \\
\hline 21 & Staphylococcus aureus (1) & 35 & 22 & 16 & - & - & - & 8 & - & - & 27 & 24 & 19 \\
\hline 22 & Staphylococcus aureus (2) & 37 & 11 & 9 & 19 & 11 & 8 & 19 & 16 & 12 & 32 & 24 & 20 \\
\hline 23 & Staphylococcus aureus (3) & 35 & 28 & 10 & 11 & 7 & - & 14 & 11 & 7 & 9 & 7 & - \\
\hline 24 & Bacillus subtilis & 35 & 20 & 18 & - & - & - & 16 & 12 & 9 & 18 & 15 & 10 \\
\hline 25 & Bacillus megaterium & 37 & 22 & 26 & 9 & - & - & 14 & 10 & 7 & 20 & 18 & 14 \\
\hline 26 & Bacillus sp.(1) & 38 & 17 & 20 & - & - & - & 15 & 13 & 9 & 11 & 9 & 7 \\
\hline 27 & Bacillus sp.(2) & 42 & 28 & 18 & - & - & - & 15 & 11 & 7 & 20 & 16 & 13 \\
\hline 28 & Bacillus sp.(3) & 35 & 25 & 14 & 19 & 14 & 10 & 24 & 20 & 14 & 30 & 27 & 21 \\
\hline 29 & Staphylococcus epidermidis & 36 & 20 & 16 & 17 & 14 & 9 & 17 & 13 & 10 & 31 & 27 & 22 \\
\hline \multirow[t]{2}{*}{30} & Enterococcus sp. & 28 & 21 & 17 & 11 & 8 & - & 24 & 19 & 14 & 30 & 25 & 20 \\
\hline & & \multicolumn{3}{|c|}{$\mathbf{H L}^{4}(\mathrm{mg} / \mathrm{mL})$} & \multicolumn{3}{|c|}{$\mathbf{H L}^{5}(\mathrm{mg} / \mathrm{mL})$} & \multicolumn{3}{|c|}{$\mathbf{H L}^{6}(\mathrm{mg} / \mathrm{mL})$} & \multicolumn{3}{|c|}{$\mathbf{H L}^{7}(\mathrm{mg} / \mathrm{mL})$} \\
\hline 1 & Escherichia coli (1) & 21 & 18 & 16 & - & - & - & - & - & - & 7 & - & - \\
\hline 2 & Escherichia coli (2) & 13 & 10 & 7 & - & - & - & - & - & - & 17 & 14 & 9 \\
\hline 3 & Escherichia coli (3) & 10 & 8 & 7 & - & - & - & 12 & 10 & 7 & - & - & - \\
\hline 4 & Escherichia coli (4) & 25 & 21 & 18 & - & - & - & - & - & - & 16 & 14 & 10 \\
\hline 5 & Pseudomonas aeruginosa (1) & 17 & 14 & 11 & - & - & - & - & - & - & 18 & 15 & 9 \\
\hline 6 & Pseudomonas aeruginosa (2) & 15 & 12 & 9 & - & - & - & - & - & - & - & - & - \\
\hline 7 & Pseudomonas aeruginosa (3) & 21 & 19 & 16 & 22 & 19 & 16 & 16 & 12 & 7 & 11 & 7 & - \\
\hline 8 & Pseudomonas sp. & 14 & 11 & 8 & - & - & - & - & - & - & - & - & - \\
\hline 9 & Pseudomonas aurantiaca & 23 & 20 & 18 & 20 & 17 & 15 & 15 & 10 & 8 & 15 & 11 & 8 \\
\hline 10 & Salmonella typhi (1) & 13 & 9 & 7 & - & - & - & - & - & - & 9 & - & - \\
\hline 11 & Salmonella typhi (2) & 22 & 19 & 16 & - & - & - & - & - & - & - & - & - \\
\hline 12 & Salmonella sp. & 19 & 18 & 16 & - & - & - & - & - & - & - & - & - \\
\hline 13 & Stenotroph maltophilia & 26 & 23 & 19 & - & - & - & - & - & - & - & - & - \\
\hline 14 & Enterobacter cloacae & 19 & 15 & 11 & - & - & - & & & & - & - & - \\
\hline 15 & Enterobacter aerogenes & 28 & 24 & 21 & - & - & - & - & - & - & - & - & - \\
\hline 16 & Klebsiella pneumonia & 15 & 10 & 7 & - & - & - & - & - & - & - & - & - \\
\hline 17 & Achromobacter xylosoxidans & 22 & 18 & 16 & - & - & - & - & - & - & - & - & - \\
\hline 18 & Azospirillum lipoferum & 14 & 12 & 9 & - & - & - & - & - & - & - & - & - \\
\hline 19 & Rhizobium sp. & 15 & 12 & 8 & - & - & - & - & - & - & - & - & - \\
\hline 20 & Citrobacter freundii & 20 & 16 & 13 & - & - & - & - & - & - & - & - & - \\
\hline 21 & Staphylococcus aureus (1) & 20 & 18 & 15 & - & - & - & - & - & - & 35 & 30 & 24 \\
\hline 22 & Staphylococcus aureus (2) & 19 & 17 & 13 & 16 & 11 & - & - & - & - & - & - & - \\
\hline 23 & Staphylococcus aureus (3) & 18 & 16 & 15 & - & - & - & - & - & - & 22 & 18 & 13 \\
\hline 24 & Bacillus subtilis & 14 & 11 & 8 & - & - & - & - & - & - & 9 & - & - \\
\hline 25 & Bacillus megaterium & 16 & 11 & 7 & - & - & - & - & - & - & 11 & 9 & - \\
\hline 26 & Bacillus sp.(1) & 16 & 13 & 9 & - & - & - & - & - & - & 10 & 8 & - \\
\hline 27 & Bacillus sp.(2) & 13 & 10 & 8 & - & - & - & - & - & - & - & - & - \\
\hline 28 & Bacillus sp.(3) & 35 & 30 & 27 & 26 & 21 & 18 & 17 & 13 & 10 & 31 & 26 & 20 \\
\hline 29 & Staphylococcus epidermidis & 20 & 18 & 14 & - & - & - & - & - & - & - & - & - \\
\hline 30 & Enterococcus sp. & 21 & 17 & 15 & - & - & - & 21 & 17 & 13 & 20 & 16 & 11 \\
\hline
\end{tabular}

Where $\mathrm{L}=$ Levofloxacin, $\mathrm{C}=$ Cefixime and $\mathrm{A}=$ Amoxylin; the blank boxes show no appreciable activity. 
respectively. It was strongly active against Pseudomonas aeruginosa (2) and (3), Pseudomonas aurantiaca, Pseudomonas aurantiaca, Salmonella typhi (1) and (2), Salmonella sp., Enterobacter cloacae, Citrobacter freundii, Staphylococcus aureus (1) and (2), Bacillus sp. (3), Staphylococcus epidermidis and Enterococcus sp. with zones of inhibition between 27-33 $\mathrm{mm}$ and MIC values between $20-40 \mu \mathrm{g} / \mathrm{mL}$. It was moderately active against rest of microbes with MIC values in the range 80-120 $\mu \mathrm{g} / \mathrm{mL}$ (Table-2).

$\mathbf{H L}^{4}$ was weakly active against Escherichia coli (3) with zone of inhibition of $10 \mathrm{~mm}$ and MIC value of $200 \mu \mathrm{g} / \mathrm{mL}$. It was strongly active against Stenotroph maltophilia, Enterobacter aerogenes and Bacillus sp. (3) with zones of inhibition between 26-33 mm and MIC values between 20-40 $\mu \mathrm{g} / \mathrm{mL}$. It was moderately active against the rest with MIC values 80-160 $\mu \mathrm{g} / \mathrm{mL}$ (Table-2).

$\mathbf{H L}^{\mathbf{5}}$ was moderately active against Pseudomonas aeruginosa (3), Pseudomonas aurantiaca, Staphylococcus aureus (2) and Bacillus sp. (3) with zones of inhibition between 16$25 \mathrm{~mm}$ and MIC values between $80-160 \mu \mathrm{g} / \mathrm{mL}$. It was totally inactive against rest of microbes (Table-2).

$\mathbf{H L}^{6}$ was weakly active against Escherichia coli (3) with zone of inhibition of $12 \mathrm{~mm}$ and MIC value of $200 \mu \mathrm{g} / \mathrm{mL}$. It showed moderate activity against Pseudomonas aeruginosa (3), Pseudomonas aurantiaca, Bacillus sp. (3) and Enterococcus $s p$. with zones of inhibition in between 15-21 mm and
MIC values between $120-160 \mu \mathrm{g} / \mathrm{mL}$. It was inactive against rest microbes (Table-2).

HL $^{7}$ was weakly active against $P$ seudomonas aeruginosa (3), Bacillus megaterium and Bacillus sp. (1) with zones of inhibition between 10-11 mm and MIC value of $200 \mathrm{~g} / \mathrm{mL}$ each. It was moderately active against Escherichia coli (2) and (4), Pseudomonas aeruginosa (1), Staphylococcus aureus (3) and Enterococcus sp. with zones of inhibition in between 17-22 $\mathrm{mm}$ and MIC range between $80-160 \mu \mathrm{g} / \mathrm{mL}$. It showed strong activity against Staphylococcus aureus (1) and Bacillus sp. (3) with zones of inhibition 35 and 31, respectively and MIC value of $20 \mu \mathrm{g} / \mathrm{mL}$ each (Table-2).

Comparing the screening results of $\mathbf{H L}^{1}$ and $\mathbf{H L}^{2}$ and $\mathbf{H L}^{\mathbf{6}}$ and $\mathbf{H L}^{7}$ gave a trend proving that $p$-substitution of benzene with azomethine group is more effective than $o$-substitution and hence are better antimicrobial agents.

$\mathbf{H L}^{4}$ was strongly active against Escherichia coli (1) and (4), Pseudomonas aeruginosa (3), Pseudomonas aurantiaca, Stenotroph maltophilia, Enterobacter aerogenes, Achromobacter xylosoxidans and Bacillus sp. (3) even in 0.5 $\mathrm{mg} / \mathrm{mL}$ concentration with zones of inhibition between 16-27 $\mathrm{mm}$.

$\mathbf{H L}^{5}$ was strongly active against Bacillus sp. (3) and Pseudomonas aeruginosa (3) in its lowest concentration with zones of inhibition $18 \mathrm{~mm}$ and $16 \mathrm{~mm}$, respectively whereas $\mathbf{H L}^{7}$ was strongly active against Staphylococcus aureus (1)

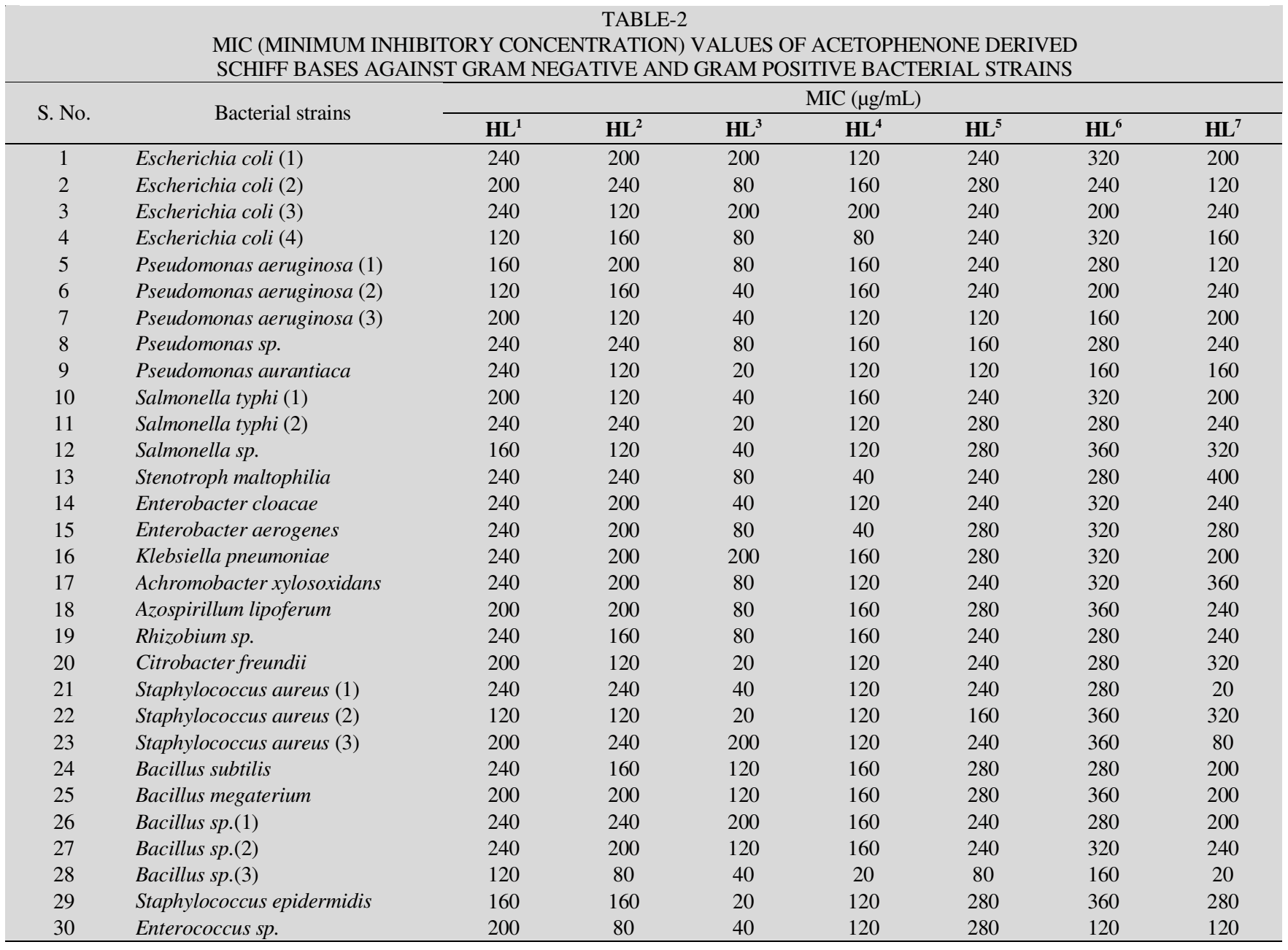


and Bacillus sp. (3) with zones of inhibition of $21 \mathrm{~mm}$ and $20 \mathrm{~mm}$.

\section{Conclusion}

Seven different acetophenone derived Schiff bases were prepared with different amines. The base (E)-N-(1-phenylethylidene)naphthalen-2-amine is being reported for the first time. The synthesized compounds were characterized by spectral study and screened against 30 different bacterial strains. $\mathrm{N}^{1}, \mathrm{~N}^{4}$-bis(1-phenylethylidene)benzene-1,4-diamine had outstanding activity against Staphylococcus aureus, thus it could be used as a hit to cure human skin and nasal passage nosocomial diseases. The new compound (E)-N-(1-phenylethylidene)naphthalen-2-amine had a remarkable versatility to combat Enterococcus sp., Citrobacter freundii, Salmonella typhi and Pseudomonas aurantiaca much better than any of reported antibiotics and hence, could be a lead molecule to prevent wide range of nosocomial infections including respiratory, urinary and blood infections and typhoid. $(E)-1$ phenyl-2-(1-phenylethylidene)hydrazine had extraordinary activity against Enterobacter aerogenes equal to that of Levofloxacin and can be formulated into drug fighting against septic arthritis, endocarditis and osteomyelitis among others, after in vivo testing. Our results confirmed the widely reported antimicrobial efficacy of the Schiff bases.

\section{REFERENCES}

1. S.B. Levy and B. Marshall, Nature Med., 10, 122 (2004).

2. L.E. Nicolle, Infection Control Programmes to contain Antimicrobial Resistance, WHO, Switzerland (2001).

3. T. Braine, Bull. World Health Organ, 89, 88 (2011).

4. Anonymous, The Top Ten Causes of Death, WHO (2011); www.who.int /mediacentre/factsheets/fs310/en/index.htmL, Accessed Sep 4 (2012).

5. D. Kumar, A. Syamal, V. Pandey and M. Rani, Asian J. Chem., 23, 5004 (2011).

6. W.C.J. Ross and G.P. Warwick, J. Chem. Soc., 1956, 1724 (1956).

7. S. Kumar, D.N. Dhar and P.N. Saxena, J. Sci. Ind. Res., 68, 181 (2009).
8. A. Prakash and D. Adhikari, Int. J. Chem. Technol. Res., 3, 1891 (2011).

9. S. Baluja and S. Chanda, J. Chem. Biol. Phys. Sci., 2, 1176 (2012).

10. S. Baluja, A. Solanki and N. Kachhadia, J. Iran. Chem. Soc., 3, 312 (2006).

11. V. Pawar, S. Joshi and V. Uma, J. Chem. Pharm. Res., 3, 169 (2011).

12. A. Jarrahpour, D. Khalili, E.D. Clercq, C. Salmi and J.M. Brunel, Molecules, 12, 1720 (2007).

13. B.H.M. Jayakumarswamy, F. Rahaman, V.K. Revankar and K.V. Pai, Int. J. Pharm. Technol. Res., 3, 1864 (2011).

14. S.M. Sondhi, M. Dinodia, S. Jain and A. Kumar, Indian J. Chem., 48B, 1128 (2009)

15. M. Jesmin, M.M. Ali and J.A. Khanam, Thai J. Pharm. Sci., 34, 20 (2010).

16. S.N. Pandeya and N. Rajput, Der Pharm. Lett., 4, 938 (2012).

17. Y. Al-Abed, L. Dubrovsky, B. Ruzsicska, M. Seepersaud and M. Bukrinsky, Bioorg. Med. Chem. Lett., 12, 3117 (2002).

18. Y.M.S.A. Al-Kahraman, H.M.F. Madkour, D. Ali, M. Yasinzai and M. Molecules, 15, 660 (2010).

19. S. Chandra and U. Kumar, Spectrochim. Acta A, 61, 219 (2005).

20. A. Prakash, B.K. Singh, N. Bhojak and D. Adhikari, Spectrochim. Acta A, 76, 356 (2010).

21. A.P. Mishra and M. Soni, Met. Based. Drugs, Article ID 875410 (2008).

22. R.R. Zaky, K.M. Ibrahim and I.M. Gabr, Spectrochim. Acta A, 81, 28 (2011).

23. N.H. Al-Shaalan, Molecules, 16, 8629 (2011).

24. W.T. Gao and Z. Zheng, Molecules, 7, 511 (2002).

25. A.H. Channar, M.Y. Khuhawar and S.W. Shah, Eur. Polym. J., 34, 133 (1998).

26. S. Samal, S. Acharya, R.K. Dey and A.R. Ray, Talanta, 57, 1075 (2002).

27. A.M. Mazloum, P. Pourhakak and N.M. Salavati, Anal. Sci., 22, 865 (2006).

28. D.S. Masterson, C. Shirley and D.T. Glatzhofer, J. Mol. Catal. A, 361, 111 (2012)

29. D. Ahmed, M.A. Chaudhary, A. Raza, A. Waheed, S.R. Khan and M. Ikram, Asian J. Chem., 24, 68 (2012).

30. CLSI, Performance Standards for Antimicrobial Disk Susceptibility Tests: Fifteenth Informational Supplement M 100-S15. CLSI, Wayne, PA, USA (2005).

31. S. Kumar, P. Kumar and N. Sati, J. Pharm. Bioallied Sci., 4, 246 (2012);

32. NCCLS, Methods for Dilution Antimicrobial Susceptibility Tests for Bacteria that Grow Aerobically, Approved Standard, M7-A6. Wayne, Pennsylvania, edn. 6 (2003).

33. G. Rajendran and K.G. Usha Devi, Asian J. Chem., 19, 223 (2007). 